\title{
Shaping the Relative Importance of Needs from Recent to Early Autobiographical Experiences
}

\author{
JOSEPH P. GREEN' ${ }^{1}$ and PATRICK J. CARROLL, The Ohio State University-Lima, Lima, OH, USA; KATHRYN A. WILLIAMS, Illinois \\ School of Professional Psychology, Chicago, IL, USA.
}

\begin{abstract}
Although past work suggests that people place greater importance of enhancement needs (e.g., self-esteem) over safety needs, recent work demonstrates that contextual factors like event valence (satisfying or distressing) can shift the importance of security above enhancement needs. This study examines whether the effect of event valence on relative need importance for recent memories can carry over to shift the importance of safety relative to enhancement needs in one's earliest childhood memories. At time 1 (T1), participants recalled their most "distressing" (securityrelevant) or "satisfying" (enhancement-relevant) experience and rated the importance of 10 needs in defining the event. Next, all participants freely recalled their earliest memory and rated the importance of different needs in defining that event. As predicted, event valence determined the importance of security relative to enhancement needs at $\mathrm{T} 1$, such that enhancement needs were more important for satisfying events whereas security needs were more important for distressing events. Moreover, as predicted, these differences in relative need importance at T1 (satisfying vs. distressing) carried over to shape relative need importance in participants' earliest memories. We close by discussing implications for human motivation.
\end{abstract}

\section{INTRODUCTION}

The study of needs has attracted psychologists for many reasons. First, needs have enormous explanatory value in that a single need can explain a wide range of behaviors. Thus, the need for relatedness can explain why people derogate out-group members to why they develop intimate relationships (Baumeister and Leary 1995). Beyond their explanatory significance, needs have practical significance in that they offer precise recommendations for what can restore health when it is lost. Just as rain and nutrients restore the dying plant, interventions that restore unfulfilled needs can promote human health and thriving supported by those needs (Ryan and Deci 2000). The appeal of needs is further enhanced by suggestions that their significance for behavior is not limited to a few isolated contexts but, instead, extends across most social, cultural, and historical contexts (Baumeister and Leary 1995; Sheldon et al. 2001). So, relatedness needs are important for health and well-being, regardless of whether one is of Inuit ancestry or a soccer mom, whether from the east or west, and, even, whether one lived long ago or now. Whereas the importance of goals or motives may change across contexts, psychologists can generally depend on the absolute importance of

${ }^{1}$ Address correspondence to Joseph P. Green, Department of Psychology, 4240 Campus Drive, The Ohio State UniversityLima, Lima, OH 45804. Email: green.301@osu.edu basic needs - across contexts - to understand and predict behavior.

Whereas the above work suggests that the absolute importance of needs may extend across most contexts, recent work suggests that the relative importance of basic needs may depend upon contextual factors (Sheldon et al. 2001). For example, certain situational factors (satisfying vs. unsatisfying events; distressing vs. satisfying events) influence the importance of security relative to enhancement (e.g., autonomy) needs even though both are vital to mental health (Kasser 2002; Sheldon and Kasser 2008; Carroll et al. 2009). Moreover, within enhancement needs, cultural factors (e.g., individualism vs. collectivism) moderate the importance of autonomy relative to relatedness even though both needs independently enhance mental health (Oishi et al. 1999). The current investigation aims to extend past work to show that contextual shifts in need importance for recent memories may bias relative need importance in the free recall (that is, no instructions to recall positive or negative memory) of earliest autobiographical memories.

First, we briefly review prior theories of needs. Next, we review work on the factors, like event valence, that influence relative need importance in recent memories. Then, we articulate the investigative purpose and predictions of the present work. Specifically, we test 
whether the effect of event valence (satisfying vs. distressing) on relative need importance for recent memories carries over to influence the relative importance of those needs for participants' earliest memories. For example, would the relatively greater importance assigned to security (vs. enhancement) needs for a recent distressing event recollection carry over to inflate the importance of security (vs. enhancement) needs for their earliest memory, even if the actual early event was not defined by security concerns?

\section{Existing Models of Human Motivation}

Traditional Need Pyramid. Maslow organized 5 needs within a pyramid of relative importance (Maslow 1954). According to Maslow, the pyramid of importance begins with basic biological (e.g., hunger) and security needs at levels 1 and 2, followed by belongingness and self-esteem at levels 3 and 4 and, ultimately, self-actualization needs at level 5 . An important feature of Maslow's pyramid is that the satisfaction of basic needs was a precondition to the pursuit of higher needs. That is, people must first satisfy basic security needs before attempting to satisfy belongingness and self-esteem needs which, in turn, must be satisfied before attempting to satisfy self-actualization (Maslow 1954).

Related to the motivational priority placed on basic over secondary higher needs, Maslow distinguished the last self-actualization need from the 4 lower needs in terms of its unique association with the enhancement of well-being rather than the minimization of distress. Specifically, Maslow defined self-actualization as a being-need (B-Need), whose satisfaction was associated with the enhancement of well-being. By contrast, he defined the 4 foundational needs as deficit-needs (D-Needs), whose deprivation was uniquely associated with psychological distress and illness (Maslow 1954). Thus, Maslow claimed that the failure to satisfy self-actualization needs would not necessarily precipitate mental distress and illness so long as the 4 foundational needs of the pyramid were satisfied. In fact, he suggested that most adults (approximately 98\%) would never experience the enhanced well-being that results from satisfying self-actualization needs, yet, still lead relatively normal lives free of mental distress so long as they could satisfy their basic needs for safety, belongingness, and self-esteem.

Limits of the Traditional Need Pyramid: Rise of the Reduced 2-Level Pyramid. Despite its intuitive appeal, the evidence has not supported the 5-step chain of motivational importance whereby people always begin by satisfying basic physiological and safety needs, before moving to esteem and belongingness needs, through to self-actualization needs (Wahbah and Bridwell 1976; Sheldon et al. 2001). Although the 5-level pyramid has garnered little empirical support, considerable evidence has amassed for an alternative 2-level pyramid, consisting of security and enhancement needs (Oishi et al. 1999; Sheldon et al. 2001). Within the reduced pyramid, the first level consists of basic "security" or "deficit" needs whereas the second level consists of "enhancement" or "growth" needs (Bowlby 1969, 1973; Wahbah and Bridwell 1976; Higgins 1997; Sheldon and Kasser 2008).

So, there are notable similarities and differences between the reduced 2-level and original 5-level model. Regarding similarities to Maslow's original model, the reduced pyramid assumes that basic physiological and security needs must be satisfied before one can pursue higher level needs (Hart et al. 2005). Moreover, these models define physiological and psychological security as deficit needs whose satisfaction predicts minimization of distress rather than the enhancement of well-being (Wahbah and Bridwell 1976).

Despite these points of similarity, the reduced and 5-level pyramid models differ on two key points. Beyond consolidating the number of levels from 5 to 2 (security vs. enhancement) needs (Bowlby 1969; Wahbah and Bridwell 1976; Higgins 1997), the reduced model also extends the classification of enhancement needs (e.g., self-esteem and relatedness) to those that Maslow originally defined as deficit (D-Needs) needs (Oishi et al. 1999). Although the precise set of enhancement needs may vary across models, most reduced hierarchies include relatedness, self-esteem, autonomy, and competence, as well as selfactualization at this second level (Sheldon et al. 2001).

Self-Determination Theory. The assumption that enhancement needs can include those that Maslow originally defined as deficit needs has gained increasing traction in other modern theories of human motivation. Self-Determination Theory (SDT) (Sheldon et al. 2001) represents just one example that exalts 3 needs other than self-actualization as enhancement needs, whose satisfaction is uniquely associated with enhanced mental health and well-being. To enhance well-being, this theory argues that people need to feel effective in their activities (competence), feel their activities are self-chosen (autonomy), and feel a sense of closeness 
with significant others (relatedness) to enhance wellbeing (Deci and Ryan 1985).

Thus, like the reduced pyramid, SDT departs from Maslow's model by extending the classification of enhancement needs beyond self-actualization to include competence, relatedness, and autonomy as intrinsic enhancement needs that are all linked to the enrichment of positive well-being rather than the minimization of psychological distress (Sheldon et al. 1996; Deci and Ryan 2000). Moreover, similar to reduced 2-level hierarchies, SDT differs from Maslow's pyramid in the sense that it has garnered extensive empirical support (Ryan and Deci 2000). Indeed, an impressive body of evidence has amassed over the past 20 years to support the unique importance of competence, autonomy, and relatedness for enhanced well-being and thriving (Deci et al. 1994; Ryan 1995; Sheldon et al. 1996; Ryan and Deci 2000).

In addition to these differences with Maslow's pyramid, SDT is unique in other ways as well. First, unlike the reduced pyramid and Maslow's original model, it does not consider security to be an innate need at all. For example, Deci and Ryan (2000, p. 325) note: "We consider safety-security not as a basic need, but as a deficit motive... it is not a need in its own right but rather is a reaction to impoverished satisfaction of true (self-determination) needs." As this quote illustrates, these theorists do not even classify security as a need, let alone a basic need, of equal or potentially greater importance than self-determination needs. Second, unlike the reduced pyramid, it does not consider any "need" (e.g., esteem) other than competence, autonomy, and relatedness to be innate enhancement needs. Although other enhancement motivations (e.g., esteem) may exist, this model focuses on competence, autonomy, and relatedness as the only basic enhancement needs that must be satisfied across social, cultural, and historical contexts to enhance well-being (Deci and Ryan 2000).

\section{Evaluating the Context(s) of Need Importance}

As noted earlier, although the importance of all needs extends across social and historical contexts, recent evidence suggests that the relative importance of different needs can shift across different contexts. For example, recent evidence shows that the relative importance of security vs. autonomy, competence, relatedness, and self-esteem needs increased when the valence of event memories shifted from satisfying (enhancement-relevant) to unsatisfying (deprivationrelevant) event recollections (Sheldon et al. 2001).

Specifically, Sheldon and colleagues directly tested whether relative need importance varied when the focused-upon event was manipulated by having participants recall either a satisfying or unsatisfying event and then complete explicit (self-report) and implicit (emotional response) measures to assess the relative importance of different needs in defining the event recollection. The explicit measure was a 30-item descriptive inventory that asked participants to rate the extent to which the presence or absence of 10 needs (e.g., autonomy, competence, relatedness, and security) represented a defining quality of the event (satisfying vs. unsatisfying) memory. For example, regarding the security need, participants in the "recall a satisfying memory" condition rated their agreement with the statement: "This event was satisfying because I felt that I was safe from threats and uncertainties." Based on explicit ratings, participants also ranked the 10 needs from least to most important.

Participants then completed an implicit measure of event-related affect in which they reported the feelings they experienced during the recalled event. The implicit measure of affect provided a supplementary measure that could sidestep potential limitations of the primary self-report measure of relative need importance (e.g., social desirability response biases). Specifically, participants completed the Positive Affect/Negative Affect Schedule (PANAS) regarding the event (Watson et al. 1988), rating the extent to which the event evoked different moods (e.g., happy, sad, scared, proud) on a scale of 1 (not at all) to 5 (very much). Composite positive and negative affect scores were computed by averaging the ratings of positive and negative affect items separately for each participant. As in prior work, Sheldon and colleagues (2001, study 3 ) showed that enhancement needs (autonomy, competence, relatedness) were higher than security needs on the explicit salience ratings for satisfying experiences. Moreover, as in prior work, results showed that the explicit salience of fulfilled autonomy, competence, and relatedness needs accounted for a significant portion of unique variance in positive affect tied to satisfying memories (all $\beta \mathrm{s}>0.23$, all $p s<0.01$ ).

Consistent with the present argument, however, these Sheldon et al. (2001) findings show that event valence (satisfying vs. unsatisfying) moderated the relative importance of security vs. enhancement needs on both explicit (salience ratings) and implicit (affect) 
measures of relative need importance. The results showed a marginal, albeit non-significant, increase in the relative importance of security vs. enhancement needs on the explicit salience ratings when participants recalled an unsatisfying vs. satisfying event experience. As importantly, on implicit affect ratings of need importance, the explicit salience ratings for deprived security needs $(r=0.50, p<0.01)$ vs. enhancement needs of autonomy $(r=0.19, p<0.01)$, competence $(r$ $=0.25, p<0.01)$, relatedness $(r=0.13, p<0.10)$, and self-esteem $(r=0.39, p<0.01)$ showed the highest correlation with negative affect ratings evoked by the unsatisfying event memories.

Finally, in a simultaneous regression model of the affect balance score in their final study (Sheldon et al. 2001), the explicit salience of deprived security needs accounted for a greater proportion of unique variance than either of the two enhancement needs (competence, $\beta=-0.22, p<0.01$; self-esteem, $\beta=-0.18, p<0.05)$ that emerged as significant predictors in the analyses. Thus, the deprivation of security vs. enhancement needs not only showed a higher correlation with the level of negative affect but also explained more unique variance in the relative intensity of negative vs. positive affect evoked by unsatisfying event memories. When taken together, the results across explicit (salience) and implicit (affect) measures suggest that event valence could potentially moderate the relative importance of enhancement vs. security needs.

Recently, Carroll and colleagues (2009) extended Sheldon's findings to provide a better test of the potential contextual effect of shifts in event valence. Specifically, Carroll et al. (2009) proposed that their "manipulation" of event valence (satisfying vs. unsatisfying) did notvary event valence at all between bad (security-relevant) vs. good (enhancement-relevant) events but, instead, only varied the positive event focus between the presence vs. absence of good (enhancement-relevant) events (see also Higgins 1997). That is, although unsatisfying events are undesired experiences, they are primarily relevant to the deprivation/fulfillment of enhancement needs rather than security needs. A valid manipulation of event valence from the presence of bad (securityrelevant) as well as good (enhancement-relevant) events would be required to truly test whether this contextual factor moderates the relative importance of security vs. enhancement needs.

Unlike unsatisfying events, distressing events are inherently relevant to security needs in that they are uniquely defined by salient cues that signal threats to security (Carroll et al. 2006). Consistent with these conceptual claims, empirical evidence indicates that certain events are primarily defined by the distressing experience of threats to security needs (Sheldon and Kasser 2008). That is, distressing events have more implications for security needs because these events are uniquely defined by salient cues signaling actual (or potential) threats to ongoing security vs. enhancement needs. Most importantly, though, this work suggests that the greater salience of threat (vs. other) cues in distressing events amplifies the importance of security over enhancement needs given the unique implications of threat for the regulation of security needs (Sheldon and Kasser 2008). In sum, Carroll et al. (2009) proposed that changes in event valence should influence the relative importance of enhancement over security needs such that security needs should assume more importance for distressing (security-relevant) memories whereas enhancement needs should assume relatively more importance when event valence shifts from distressing (security-relevant) to satisfying (enhancement-relevant) event memories.

Across two studies, Carroll et al. (2009) adopted the same general method used by Sheldon and colleagues (2001), including the explicit 30-item need inventory as well as the implicit affect measure. However, Carroll et al. (2009) modified the recollection exercise to replace Sheldon's original manipulation with a full manipulation of event valence from distressing (security-relevant) to satisfying (enhancement-relevant) memories. With respect to event valence, the relative importance of enhancement vs. security needs shifted with changes in event valence: security vs. enhancement needs were significantly more important for securityrelevant (distressing) events whereas enhancement vs. security needs became significantly more important when event valence shifted from security-relevant to enhancement-relevant (satisfying) event memories.

Of note, these findings also suggest that prior traumatic experience of hurricane survivors vs. control participants qualified the general tendency to shift significantly more importance to enhancement over security needs as event valence shifted from security-relevant to enhancement-relevant (satisfying) memories (Carroll et al. 2009). For security-relevant memories, the traumatized and non-traumatized samples in the Carroll et al. (2009) study did not differ on ratings of relative need importance as all participants rated security as significantly more important than all enhancement needs in defining 
distressing event experiences. However, when event valence shifted back to enhancement-relevant memories, only the non-traumatized control sample rated security as significantly less important than individual enhancement needs. By contrast, the traumatized sample showed no differences in the importance assigned to security relative to individual enhancement needs. Beyond moderating the general shift in relative importance in security vs. enhancement needs across security and enhancement-relevant memories, prior trauma also qualified the relative importance among different enhancement needs. Specifically, whereas individual enhancement needs of autonomy, competence, and self-esteem assumed the greatest importance in the non-traumatized sample, the interpersonal enhancement need of relatedness assumed significantly greater importance than all individual enhancement needs and security in the traumatized sample for enhancement-relevant memories. Thus, for traumatized participants, the defining feature of satisfying memories was not whether one felt competent, autonomous, or even worthy. Rather, it was whether one felt connected to important loved ones and friends.

To summarize, the findings of Carroll et al. (2009) suggest that traumatic experiences may qualify the effect of changes in event valence on relative need importance. With the exception of relatedness, traumatized (unlike non-traumatized) participants did not rate enhancement needs as more important than security needs when event valence shifts to enhancement-relevant events that offer more viable opportunities to satisfy enhancement vs. security needs.

\section{Overview of Present Work}

Prior work has identified situational (event valence) as well as dispositional (prior trauma) influences on the relative importance of different needs. The purpose of this investigation is two-fold. The first goal was to replicate the effect of changes in event valence on the importance of individual enhancement relative to security and the interpersonal enhancement need of relatedness. Specifically, we predicted that security and relatedness would assume greater importance than autonomy, competence, and self-esteem across both implicit (affect) and explicit (self-reported salience) measures for security-relevant (distressing vs. satisfying) events whereas individual enhancement needs would assume greater importance for enhancement-relevant (satisfying vs. distressing) events.
In addition, though, the second purpose was to extend past work by showing how the influence of shifts in event valence on relative need importance for recent memories might bias the subsequent recollection of earlier life memories. Specifically, we anticipated that the shifts in relative need importance produced by the manipulation of event valence (satisfying vs. distressing) for a recent memory at time 1 (T1) would carry over to shape the relative need importance of free recollections of earliest life memories at time 2 (T2). Specifically, we predicted that the relatively greater salience of security and relatedness produced by recollections of recent distressing events would bias the recollection of participants' earliest life memories to inflate the importance (salience) of security/relatedness needs to the neglect of individual enhancement needs. Conversely, we predicted that the greater importance of individual enhancement (vs. security or relatedness) produced by enhancement-relevant (satisfying) event memories would subsequently bias the free recollection of participants' earliest life memory to inflate the importance (salience) of individual enhancement needs and, in particular, the earliest individual enhancement need of hedonic pleasure (over security/relatedness) needs.

\section{Participants}

\section{MATERIALS AND METHODS}

A total of 177 undergraduate students enrolled at The Ohio State University at Lima participated in the study. Final data analyses were conducted on $N=164$ participants $\left(n_{\text {female }}=100 ; n_{\text {male }}=64 ; M_{\text {age }}=22.06, S D=\right.$ 5.95) who provided complete data across our measures.

\section{Materials}

Explicit Need Importance Inventory (NII). To explicitly assess the relative importance of psychological needs, we used a modified version of Sheldon et al.'s (2001) inventory of psychological needs. Based on theoretical and empirical work, Sheldon and colleagues instructed participants to briefly write out the details of a satisfying or unsatisfying experience. Participants then rated 30 descriptive statements reflecting 10 psychological needs (e.g., I felt that this event was satisfying (unsatisfying) because I was (was not) free to do things my own way). Response options for each item ranged from a 1 (not at all the reason) to 5 (very much the reason). Salience scores for each candidate need was based on averaging the scores across three items relevant to each individual need. Previous work 
showed adequate inter-item consistency across the 10 needs ( $\alpha>0.75$; see Carroll et al. 2009). Consistent with Sheldon et al.'s (2001) organization of needs, we averaged responses across the autonomy, relatedness, competence, and self-esteem items to create an overall "enhancement" needs index.

In an effort to broaden the event valence, Carroll et al. (2009) instructed participants to recall a "satisfying" or "distressing" event. Similarly, we instructed participants to recall the "single most personally satisfying" or "single most personally distressing" event. Unlike Sheldon et al.'s (2001) or Carroll et al.'s (2009) instructions to recall a relatively recent event (i.e., within the past week, few weeks, or past couple of months), we allowed participants to select their "single most personally satisfying" or "single most personally distressing" experience within the past five years. This allowed greater leeway for participants to select an event with high emotional impact.

Positive and Negative Affect Scale (PANAS) (Watson et al. 1988). The PANAS consists of 10 positive mood adjectives (e.g., enthusiastic, active, and alert) and 10 negative mood adjectives (e.g., distress, anger, sadness, and lethargy). In this study, participants rated how they currently felt (i.e., "right now") using the following scale: 1 = very slightly or not at all, $2=a$ little, $3=$ moderately, $4=$ quite a bit, $5=$ extremely. Positive and negative subscale scores were computed by averaging the responses across the positive and negative adjectives, respectively. Excellent inter-item consistency and test-retest reliability across the subscales have been reported (see Watson et al. 1988; Carroll et al. 2009).

Event Ratings. With the following questions, we assessed the intensity, impact, and feeling associated with the memories recalled: 1 . How intense or vivid was the event recalled? ( $1=$ not at all intenselvivid, 3 = somewhat intense/vivid, 5 = very intense/vivid); 2 . To what degree did the event recalled impact your life? $(1=$ not at all, 3 = somewhat, $5=$ very much $) ; 3$. How does the event recalled feel to you right now? ( $1=$ very distressing, 2 = distressing, 3 = a little distressing, 4 = neutral, $5=$ a little satisfying, $6=$ satisfying, $7=$ very satisfying). When instructed to recall their very earliest memory, we asked participants to indicate their age (in months) at the time of the event.

\section{Procedure}

"Satisfying" and "distressing" protocol booklets were randomly assembled and distributed to participants within groups ranging in size from 10 to 55 . We informed participants that they would recall two events from their life: one relatively recent event and another from the distant past. Participants were instructed to select an event that they felt comfortable "thinking about, writing out in detail, and answering questions about." The stated purpose of the study was to "better understand how emotional states might influence recall and how recalling certain events may reflect psychological needs." Participants were informed that they could stop or withdraw at any point during the study. They received course extra credit for their participation.

After completing a demographic information page, participants were asked to bring to mind the "single most satisfying (or distressing) event that you experienced over the past five years." Participants were instructed to interpret "satisfying" or "distressing" in "whatever way makes sense to you." We told participants that we were "being a bit vague on purpose and that they should use their own definition." We concluded the instructions by reminding participants to select a "very impactful" experience and that they be "as specific as possible" when reporting the event. Participants were given five minutes to write out the event in detail.

When the writing period ended, participants were instructed to complete the NII, PANAS, and the Event Ratings. When everyone had finished, they were then told to think about their "very earliest memory." Participants were asked to select a remembered event that "you believe that you really remember happening as the event happened and not something that you learned about later in life through family stories or by watching a video of yourself when you were younger." Participants were given five minutes to write out the details of the event constituting their earliest memory, again with the instructions to be as specific as possible. Afterwards, participants once again completed the NII, PANAS, and the Event Ratings. Participants were debriefed at the end of the study. 


\section{Preliminary Analyses}

\section{RESULTS}

The proportion of males (39\%) to females (61\%) within each of the two conditions (i.e., satisfying vs. distressing) was identical, $\mathrm{X}^{2}(1 ; N=164)=0.00, p=$ 1.00 . The age of participants $\left(M_{\text {overall }}=22.06, S D=\right.$ 5.94) did not statistically differ by condition, $t(162)$ $=0.28, p=0.78$.

\section{Primary Analyses}

\section{First Recall Attempt: Trial 1}

Event Rating Items. Responses to the Event Ratings served as a manipulation check. Consistent with the instructions for both conditions to recall an event that was very impactful and emotionally salient (e.g., recall the "single most personally satisfying or distressing event"), no differences were observed between the "satisfying" and "distressing" conditions on the Event Ratings of intensity or impactfulness of the event recalled, $F_{s}(3,160)<1.09$, ps $>0.05$. When asked how the recalled event feels right now, the average score from participants in the "satisfying" condition fell between the anchors of satisfying and very satisfying. In contrast, the average score from participants in the "distressing" condition fell between a little distressing and neutral, $F(3,160)=170.98, p<0.001$. Event Rating means and standard deviations are listed in the left-hand section of Table 1 under the Directed recall (Trial 1) heading.

We conducted a multivariate analysis of variance (MANOVA) by condition across participants' ratings of the 10 individual needs following their first recollection. The multivariate effect was significant, $F(10,153)=$ $17.40, p<0.001$. Means and standard deviations of the individual needs by condition are presented in Table 2 .

We next contrasted the relative salience of security and enhancement needs (e.g., autonomy, competence, relatedness, and self-esteem) at time 1 (T1) for the "satisfying" and "distressing" memories in a series of dependent samples t-tests (Table 3). As in prior work (Carroll et al. 2009), participants recalling a "distressing" event ranked security as their most pressing need. Consistent with predictions, participants recalling a distressing event rated security significantly higher on the explicit T1 salience scores than any of the individual enhancement needs (i.e., self-esteem, competence, autonomy), all ts (86) $>4.91$, ps $<0.001$. As in prior work (see Carroll et al. 2009), participants recalling a "satisfying" event ranked the individual enhancement need of self-esteem as their most pressing need. Consistent with predictions, moreover, participants in the satisfying recall condition rated all individual enhancement needs significantly higher on the explicit T1 salience scores than security, all ts (77)

Table 1

Event ratings, age of earliest memory, and PANAS scores across the recall trials

\begin{tabular}{|c|c|c|c|c|c|c|c|c|c|c|c|c|}
\hline & \multicolumn{4}{|c|}{$\begin{array}{l}\text { Directed recall } \\
\text { (Trial 1) }\end{array}$} & \multicolumn{4}{|c|}{$\begin{array}{l}\text { Earliest memory } \\
\text { (Trial 2) }\end{array}$} & \multicolumn{4}{|c|}{$\begin{array}{l}\text { Self-determined groups } \\
\text { (Trial 2) }\end{array}$} \\
\hline & \multicolumn{2}{|c|}{ Satisfying } & \multicolumn{2}{|c|}{ Distressing } & \multicolumn{2}{|c|}{ Satisfying } & \multicolumn{2}{|c|}{ Distressing } & \multicolumn{2}{|c|}{ Satisfying } & \multicolumn{2}{|c|}{$\overline{\text { Distressing }}$} \\
\hline & $M$ & (SD) & $M$ & $(S D)$ & $M$ & (SD) & $M$ & $(S D)$ & $M$ & (SD) & $M$ & $(S D)$ \\
\hline \multicolumn{13}{|l|}{ Event Ratings } \\
\hline Intensity & 4.13 & $(0.85)$ & 4.28 & $(0.94)$ & 3.47 & $(1.01)$ & 3.40 & (1.11) & 3.50 & $(1.02)$ & 3.74 & (1.03) \\
\hline Impactfulness & 4.27 & $(1.03)$ & 4.39 & $(1.01)$ & 2.66 & $(1.41)$ & 2.61 & $(1.37)$ & 2.95 & $(1.34)$ & 3.39 & $(1.28)$ \\
\hline Feel & 6.22 & $(0.95)$ & 3.39 & $(1.67)$ & 4.44 & $(1.37)$ & 4.46 & $(1.38)$ & 5.92 & $(0.71)$ & 2.54 & $(0.72)$ \\
\hline Age (in months)^ & \multicolumn{2}{|c|}{$\mathrm{n} / \mathrm{a}$} & \multicolumn{2}{|c|}{$\mathrm{n} / \mathrm{a}$} & 50.30 & $(20.91)$ & 59.67 & (44.68) & 59.87 & $(45.52)$ & 55.13 & $(30.20)$ \\
\hline \multicolumn{13}{|l|}{ PANAS } \\
\hline Positive subscale & 29.21 & (9.97) & 26.18 & $(9.61)$ & 23.73 & $(9.84)$ & 26.40 & (10.64) & 29.10 & (10.05) & 22.48 & $(10.05)$ \\
\hline Negative subscale & 15.34 & $(5.20)$ & 18.53 & $(7.94)$ & 14.65 & $(6.09)$ & 15.28 & $(6.58)$ & 14.08 & $(5.57)$ & 20.03 & $(8.80)$ \\
\hline \multicolumn{13}{|c|}{$\begin{array}{l}\text { Note: Within recall trials/groupings, means in bold significantly differed from one another. } \\
N=164 \text { for the Directed and Earliest Memory trials. A total of } n=93 \text { participants comprised the Self-Determined } \\
\text { groups (based on subjective ratings to the question: "How does the event recalled feel to you right now?"); } n_{\text {satisfying }}=62 ; \\
n_{\text {distressing }}=31 \text {. } \\
\wedge \text { Purported age (in months) of participants when the event constituting their earliest memory occurred. }\end{array}$} \\
\hline
\end{tabular}


$>6.52$, all $p$ s $<0.01$. Table 3 details the rankings of each need within condition for the directed recall trail.

Scores on the PANAS. Not surprisingly, participants instructed to recall a "satisfying" event within the past five years scored higher on the positive affect subscale of the PANAS than did those in the "distressing" condition, $F(2,161)=3.90, p=0.05$. They also scored lower on the negative affect subscale of the PANAS compared to those in the "distressing" condition, $F(2$, $161)=9.01, p<0.005$ (see the Directed recall (Trial 1) heading in Table 1$)$.

Table 2

Comparison of mean ratings for each need by condition (Trial 1)

\begin{tabular}{|c|c|c|c|c|c|c|}
\hline & \multicolumn{2}{|c|}{ Satisfying $(n=77)$} & \multicolumn{2}{|c|}{ Distressing $(n=87)$} & \multirow[b]{2}{*}{$F$} & \multirow[b]{2}{*}{$p$} \\
\hline & Mean & $(S D)$ & Mean & $(S D)$ & & \\
\hline Autonomy & 3.77 & $(1.03)$ & 2.08 & $(1.05)$ & 107.23 & .000 \\
\hline Competence & 3.72 & $(1.29)$ & 1.85 & $(1.02)$ & 107.39 & .000 \\
\hline Relatedness & 3.79 & $(1.26)$ & 2.32 & $(1.27)$ & 55.09 & .000 \\
\hline Self-Actualization & 3.64 & $(1.17)$ & 2.00 & $(1.16)$ & 80.27 & .000 \\
\hline Physical & 2.36 & $(1.21)$ & 1.89 & $(1.14)$ & 6.64 & .011 \\
\hline Pleasure & 2.93 & $(1.07)$ & 1.77 & $(0.98)$ & 52.13 & .000 \\
\hline Money & 1.86 & $(1.10)$ & 1.35 & $(0.82)$ & 11.38 & .001 \\
\hline Security & 2.63 & $(1.18)$ & 2.89 & $(1.31)$ & 1.78 & .184 \\
\hline Self-Esteem & 3.87 & $(1.12)$ & 2.13 & $(1.24)$ & 87.31 & .000 \\
\hline Popularity & 2.68 & $(1.31)$ & 1.64 & $(0.93)$ & 35.21 & .000 \\
\hline Enhancement index & 3.79 & $(0.80)$ & 2.10 & $(0.92)$ & 154.41 & .000 \\
\hline
\end{tabular}

Table 3

Rankings of need salience within condition (Trial 1)

\begin{tabular}{|c|c|c|c|c|c|}
\hline \multicolumn{3}{|c|}{ Satisfying $(n=77)$} & \multicolumn{3}{|c|}{ Distressing $(n=87)$} \\
\hline Need Rank & Mean & (SD) & Need Rank & Mean & (SD) \\
\hline Self-Esteem & 3.87 & $(1.12)^{\mathrm{a}}$ & Security & 2.89 & $(1.31)^{\mathrm{a}}$ \\
\hline Relatedness & 3.79 & $(1.26)^{\mathrm{a}}$ & Relatedness & 2.32 & $(1.27)^{\mathrm{a}, \mathrm{b}}$ \\
\hline Autonomy & 3.77 & $(1.03)^{\mathrm{a}}$ & Self-Esteem & 2.13 & $(1.24)^{\mathrm{b}, \mathrm{c}}$ \\
\hline Competence & 3.72 & $(1.29)^{a}$ & Autonomy & 2.08 & $(1.05)^{b, c, d}$ \\
\hline Self-Actualization & 3.64 & $(1.17)^{\mathrm{a}}$ & Self-Actualization & 2.00 & $(1.16)^{b, c, d, e}$ \\
\hline Pleasure & 2.93 & $(1.07)^{\mathrm{b}}$ & Physical & 1.89 & $(1.14)^{b, c, d, e, f}$ \\
\hline Popularity & 2.68 & $(1.31)^{b, c}$ & Competence & 1.85 & $(1.02)^{\mathrm{d}, e, f, g}$ \\
\hline Security & 2.63 & $(1.18)^{\mathrm{b}, \mathrm{c}, \mathrm{d}}$ & Pleasure & 1.77 & $(0.98)^{\mathrm{e}, \mathrm{f}, \mathrm{g}, \mathrm{h}}$ \\
\hline Physical & 2.36 & $(1.21)^{\mathrm{c,d}}$ & Popularity & 1.64 & $(0.93)^{\mathrm{f}, \mathrm{g}, \mathrm{h}}$ \\
\hline Money & 1.86 & $(1.10)^{\mathrm{e}}$ & Money & 1.35 & $(0.82)^{\mathrm{i}}$ \\
\hline
\end{tabular}




\section{Second Recall Attempt: Trial 2}

Participants who originally were instructed to recall either a "satisfying" or "distressing" event were later instructed to recall their very earliest memory without any additional instruction regarding event valence. Our groups, classified on the basis of the initial "satisfying" and "distressing" recall conditions, evaluated their earliest memories similarly across the Event Rating items and how old they were when the event recalled purportedly occurred $\left[F_{\mathrm{S}}(4,159)<2.83\right.$, $p s>0.09]$, and on the positive and negative subscales of the PANAS $\left[F_{\mathrm{S}}(2,90)<2.77, p s>0.09\right]$. Means and standard deviations are listed in the middle section of Table 1 under the Earliest memory (Trial 2) heading.

Needs Associated with Earliest Memory Reports. In order to explore whether the first recall trial-with specific instructions to recall either a "satisfying" or "distressing" event-primed or influenced the nature of the earliest memories, we examined the needs associated with the earliest memories as a function of participants' originally assigned condition. We conducted a multivariate analysis of variance (MANOVA) by condition across participants' ratings of the 10 individual needs following the writing of their earliest memories. The multivariate effect failed to reach significance, $F(10,153)=1.80, p=0.64$. Means and standard deviations of the individual needs by condition for the second recall (Trial 2) are presented in Table 4.

We next contrasted the relative salience of needs for the "satisfying" and "distressing" conditions in a series of paired samples t-tests (see Table 5). Consistent with predictions, the results of a dependent sample t-test showed that the relatively greater salience of security over individual enhancement needs in recollections of recent distressing (vs. satisfying) memories carried over to inflate the relative salience of security over individual enhancement needs in the free recollection of participants' earliest life memories at time 2 (T2), $t=$ $1.97, p=0.05$. Moreover, consistent with predictions, regression analyses revealed that the relative salience of security over enhancement needs at time 1 (T1) uniquely predicted the relative salience of security over enhancement needs in the ultimate free recollections of one's earliest life memories at T2, $b=0.17, s e=0.07$, $t=2.46, p=0.01$.

Despite finding support for our predictions on the relative salience measures, it is worth noting that the absolute salience of security needs among those in the distressing condition actually diminished from $\mathrm{T} 1$ $(M=2.89)$ to $\mathrm{T} 2(M=2.59)$. Although the absolute salience of security dropped from $\mathrm{T} 1$ to $\mathrm{T} 2$ in the distressing condition, the salience of the interpersonal enhancement need of relatedness significantly increased

Table 4

Comparison of mean ratings for each need by condition (Trial 2)*

\begin{tabular}{|c|c|c|c|c|c|c|}
\hline & \multicolumn{2}{|c|}{ Satisfying $(n=77)$} & \multicolumn{2}{|c|}{ Distressing $(n=87)$} & \multirow[b]{2}{*}{$F$} & \multirow[b]{2}{*}{$p$} \\
\hline & Mean & (SD) & Mean & (SD) & & \\
\hline Autonomy & 2.39 & $(1.15)$ & 2.40 & $(1.23)$ & 0.00 & .96 \\
\hline Competence & 1.97 & $(1.05)$ & 2.31 & $(1.26)$ & 3.30 & .07 \\
\hline Relatedness & 3.40 & $(1.26)$ & 3.25 & $(1.27)$ & 0.57 & .45 \\
\hline Self-Actualization & 1.68 & $(0.92)$ & 1.85 & $(1.03)$ & 1.12 & .29 \\
\hline Physical & 1.82 & $(0.95)$ & 2.05 & $(1.11)$ & 1.88 & .17 \\
\hline Pleasure & 2.42 & $(1.11)$ & 2.66 & $(1.42)$ & 1.41 & .24 \\
\hline Money & 1.44 & $(0.61)$ & 1.60 & $(0.73)$ & 2.41 & .12 \\
\hline Security & 2.36 & $(1.32)$ & 2.59 & $(1.23)$ & 1.28 & .26 \\
\hline Self-Esteem & 2.29 & $(1.19)$ & 2.46 & $(1.38)$ & 0.70 & .40 \\
\hline Popularity & 1.76 & $(0.95)$ & 1.72 & $(0.84)$ & 0.07 & .79 \\
\hline Enhancement index & 2.52 & $(0.90)$ & 2.56 & $(0.96)$ & 0.36 & .55 \\
\hline
\end{tabular}

Note: Enhancement index is the average score across autonomy, relatedness, competence, and self-esteem items.

* Both groups received a positively worded need inventory booklet (i.e., administered to "satisfying" participants in Trial 1). 
Table 5

Rankings of need salience within condition ("earliest memory"/Trial 2)

\begin{tabular}{|c|c|c|c|c|c|}
\hline \multicolumn{3}{|c|}{ Satisfying $(n=77)$} & \multicolumn{3}{|c|}{ Distressing $(n=87)$} \\
\hline Need Rank & Mean & $(S D)$ & Need Rank & Mean & (SD) \\
\hline Relatedness & 3.40 & $(1.26)^{\mathrm{a}}$ & Relatedness & 3.25 & $(1.27)^{\mathrm{a}}$ \\
\hline Pleasure & 2.42 & $(1.11)^{\mathrm{b}}$ & Pleasure & 2.66 & $(1.42)^{\mathrm{b}}$ \\
\hline Autonomy & 2.39 & $(1.15)^{\mathrm{b}, \mathrm{c}}$ & Security & 2.59 & $(1.23)^{\mathrm{b}}$ \\
\hline Security & 2.36 & $(1.32)^{b, c, d}$ & Self-Esteem & 2.46 & $(1.38)^{\mathrm{b}}$ \\
\hline Self-Esteem & 2.29 & $(1.19)^{\mathrm{b}, \mathrm{c}, \mathrm{d}}$ & Autonomy & 2.40 & $(1.23)^{\mathrm{b}, \mathrm{c}}$ \\
\hline Competence & 1.97 & $(1.05)^{\mathrm{d}}$ & Competence & 2.31 & $(1.26)^{\mathrm{b}, \mathrm{d}}$ \\
\hline Physical & 1.82 & $(0.95)^{\mathrm{d}, \mathrm{e}}$ & Physical & 2.05 & $(1.11)^{\mathrm{c}, \mathrm{d}, \mathrm{e}}$ \\
\hline Popularity & 1.76 & $(0.95)^{d, e}$ & Self-Actualization & 1.85 & $(1.03)^{\mathrm{e}, \mathrm{f}}$ \\
\hline Self-Actualization & 1.68 & $(0.92)^{e, f}$ & Popularity & 1.72 & $(0.84)^{e, f}$ \\
\hline Money & 1.44 & $(0.61)^{f}$ & Money & 1.60 & $(0.73)^{\mathrm{f}}$ \\
\hline
\end{tabular}

from T1 $(M=2.32)$ to the final T2 $(M=3.25)$. Moreover, although both groups assigned significantly greater importance to relatedness compared to all other needs at T2, participants in the satisfyinggroup - unlike the distressing group — actually showed a decline in the absolute salience of relatedness from T1 $(M=3.79)$ to T2 $(M=3.40)$.

Although we generally supported the predictions regarding the generalization of relative need importance across time points in the distressing condition, the findings did not support the prediction that those participants in the satisfying (vs. distressing) condition would generalize the relatively greater importance of individual enhancement over security needs from $\mathrm{T} 1$ to $\mathrm{T} 2$, all $t s<-0.34$, all $p s>0.73$. Although the results did not support the predicted generalization of importance for individual enhancement needs, these participants did assign significantly greater importance to the interpersonal enhancement need of relatedness than all other needs, including security, all $t \mathrm{~s}>6.31$, all $p$ s $<0.01$. Moreover, although only marginally significant, regression analyses revealed that the relative salience of relatedness over security needs at $\mathrm{T} 1$ uniquely predicted the relative salience of relatedness over security in the ultimate free recollections of one's earliest life memories at T2, $b=0.18, s e=0.09, t=1.79, p<0.07$. Thus, although we did not support the generalization of greater importance for the individual enhancement over security needs, results showed that the greater importance of the interpersonal enhancement need of relatedness over security at $\mathrm{T} 1$ generalized to the free recollection of one's earliest autobiographical memory. We return to this point in the general discussion.

\section{Self-Determined Groups: Second Recall Attempt}

Event Ratings and PANAS Scores. We created two extreme groups based on how participants reported feeling after thinking about the event constituting their earliest memory and reanalyzed data following the second recall attempt based on these new groupings. Participants who reported feeling distressed or very distressed ( $\leq 2$ on the feel item) about their earliest memories comprised the Self-Determined Distressing group $(n=31)$; whereas, those who reported feeling satisfied or very satisfied ( $\geq 6$ on the feelitem) comprised the Self-Determined Satisfying group $(n=62)$. Using these groupings, participants' ratings of the intensity and impactfulness of their earliest memories did not differ, nor did they differ in terms of their reported age when the event occurred, $F_{\mathrm{S}}(1,88)<2.25$, $p$ s $>0.14$. Not surprisingly, the groups significantly differed on the feel item, which served to classify the groups, $F(1$, $88)=461.83, p<0.001$. Means and standard deviations are listed in the right-hand section of Table 1 under the Self-Determined Groups (Trial 2) heading. As can also be seen in the table, and as we predicted, those reporting that they felt satisfied thinking about their earliest memory had higher positive scale scores and lower negative scale scores on the PANAS, relative to 
those who reported feeling distressed about the event, $F_{\mathrm{s}}(1,90)>8.94$, $p$ s $<0.005$.

Using these self-determined groupings, we once again conducted a multivariate analysis of variance (MANOVA) across participants' ratings of the 10 individual needs. The multivariate effect was significant, $F(10,82)=6.98, p<0.001$. Table 6 and Table 7 display the means and standard deviations of the individual needs, and the rankings of need salience by our self-determined groups, respectively.

Table 6

Comparison of mean ratings for each need by self-determined groups ("earliest memory"/Trial 2)*

\begin{tabular}{|c|c|c|c|c|c|c|}
\hline & \multicolumn{2}{|c|}{ Satisfying $(n=62)$} & \multicolumn{2}{|c|}{ Distressing $(n=31)$} & \multirow[b]{2}{*}{$F$} & \multirow[b]{2}{*}{$p$} \\
\hline & Mean & $(S D)$ & Mean & $(S D)$ & & \\
\hline Autonomy & 3.07 & (1.09) & 1.86 & $(1.06)$ & 25.97 & .000 \\
\hline Competence & 2.52 & $(1.24)$ & 1.83 & $(1.05)$ & 6.98 & .010 \\
\hline Relatedness & 4.02 & $(0.92)$ & 2.85 & (1.19) & 27.37 & .000 \\
\hline Self-Actualization & 2.14 & $(1.03)$ & 1.47 & $(0.69)$ & 10.69 & .002 \\
\hline Physical & 2.31 & $(1.17)$ & 1.56 & $(0.84)$ & 9.98 & .002 \\
\hline Pleasure & 3.20 & $(1.28)$ & 1.95 & $(1.01)$ & 22.88 & .000 \\
\hline Money & 1.75 & $(0.78)$ & 1.31 & $(0.58)$ & 7.80 & .006 \\
\hline Security & 3.13 & $(1.15)$ & 1.54 & $(0.84)$ & 46.79 & .000 \\
\hline Self-Esteem & 3.10 & $(1.31)$ & 1.76 & $(0.93)$ & 25.85 & .000 \\
\hline Popularity & 1.88 & $(1.00)$ & 1.59 & $(0.72)$ & 2.07 & .154 \\
\hline Enhancement index & 3.18 & $(0.77)$ & 2.07 & $(0.83)$ & 39.93 & .000 \\
\hline
\end{tabular}

Table 7

Rankings of need salience within self-determined groupings ("earliest memory"/Trial 2)

\begin{tabular}{|c|c|c|c|c|c|}
\hline \multicolumn{3}{|c|}{ Satisfying $(n=62)$} & \multicolumn{3}{|c|}{ Distressing $(n=31)$} \\
\hline Need Rank & Mean & (SD) & Need Rank & Mean & $(S D)$ \\
\hline Relatedness & 4.02 & $(0.92)^{a}$ & Relatedness & 2.85 & $(1.19)^{\mathrm{a}}$ \\
\hline Pleasure & 3.20 & $(1.28)^{\mathrm{b}}$ & Pleasure & 1.95 & $(1.01)^{\mathrm{b}}$ \\
\hline Security & 3.13 & $(1.15)^{b, c}$ & Autonomy & 1.86 & $(1.06)^{b, c}$ \\
\hline Self-Esteem & 3.10 & $(1.31)^{\mathrm{b}, \mathrm{c}, \mathrm{d}}$ & Competence & 1.83 & $(1.05)^{b, c, d}$ \\
\hline Autonomy & 3.07 & $(1.09)^{b, c, d}$ & Self-Esteem & 1.76 & $(0.93)^{b, c, d, e}$ \\
\hline Competence & 2.52 & $(1.24)^{\mathrm{e}}$ & Popularity & 1.59 & $(0.72)^{b, c, d, e}$ \\
\hline Physical & 2.31 & $(1.17)^{\mathrm{e}, \mathrm{f}}$ & Physical & 1.56 & $(0.84)^{b, c, d, e}$ \\
\hline Self-Actualization & 2.14 & $(1.03)^{\mathrm{e}, \mathrm{f}, \mathrm{g}}$ & Security & 1.54 & $(0.84)^{b, c, d, e}$ \\
\hline Popularity & 1.88 & $(1.00)^{\mathrm{g}, \mathrm{h}}$ & Self-Actualization & 1.47 & $(0.69)^{c, d, e}$ \\
\hline Money & 1.75 & $(0.78)^{\mathrm{h}}$ & Money & 1.31 & $(0.58)^{\mathrm{d}}$ \\
\hline
\end{tabular}




\section{DISCUSSION}

Consistent with past work and predictions, we replicated the effect of changes in event valence on the relative importance of security and relatedness vs. individual enhancement needs. Specifically, security and relatedness assumed greater importance than individual enhancement needs at $\mathrm{T} 1$ when participants recalled a recent distressing (vs. satisfying) event experience; however, self-esteem, competence, and autonomy assumed greater importance than security at time 1 when participants recalled a recent satisfying (vs. distressing) event experience. Moreover, the findings primarily supported the novel prediction that-on a subsequent free recollection-the changes on relative need importance evoked by recollections of more recent distressing or satisfying experience would bias the subsequent recollection of participants' earliest life memory to fit the relative importance of security/ relatedness compared to enhancement needs for the recent memory.

Specifically, our results showed that the relatively greater importance of security over enhancement needs, among participants who first recalled a distressing (vs. satisfying) experience, generalized to relatively greater importance assigned to security over enhancement needs in earliest autobiographical memories. Furthermore, although the relatively greater T1 salience of individual enhancement needs did not generalize to T2, the findings did support our prediction that the relatively greater salience of the interpersonal enhancement need of relatedness did generalize to T2 when participants recalled a recent satisfying experience at $\mathrm{T} 1$.

Of course, prior work on the socialization of selfstrivings has suggested that one must first establish a stable social network before pursuing individual enhancement needs (see Baumeister and Leary 1995). For example, it is very difficult for a younger child to pursue self-esteem, autonomy, and competence given their primary focus on establishing nurturing and stable relational attachments early in life. Thus, it may be that the greater importance only generalized to the interpersonal enhancement need of relatedness simply because that is really the only enhancement need "on the block" early in life. In fact, participants rated the relative salience of needs for their earliest (childhood) autobiographical memories when the interpersonal enhancement need of relatedness still dominated over individual enhancement needs. Thus, for early memories, it seems as though the most important enhancement need is not self-esteem, competence, or autonomy but, instead, the interpersonal need of feelings of relatedness and connection to important others (e.g., parents).

It is also worth noting that the emergence of relatedness as the strongest enhancement need in earliest autobiographical memories mirrors the emergence of relatedness as the strongest enhancement need among traumatized (vs. control) participants in prior work (Carroll et al. 2009). The authors reasoned that the greater importance assigned to relatedness likely reflects the fact that trauma survivors must first rebuild their connection to a stable social environment (e.g., moving back home from a temporary Red Cross shelter) before they can pursue higher enhancement needs of building esteem, autonomy, and competence within the context of renewed social relationships. Thus, taken together with past work, the present findings suggest that anything that temporarily diminishes or disrupts the experience or felt connection to one's established social context will enhance the relative importance of interpersonal relatedness over all other individual enhancement needs. Moreover, future work could test whether the heightened importance of relatedness concerns persists until one's experience of a stable social context is restored.

Although the salience of security actually dropped from $\mathrm{T} 1$ to $\mathrm{T} 2$ in the distressing condition, the relative salience of the interpersonal enhancement need of relatedness significantly increased over individual enhancement needs from $\mathrm{T} 1$ to the final T2. Of course, an important point worth noting regards the countervailing decline in security salience as the salience of relatedness needs increased from $\mathrm{T} 1$ to $\mathrm{T} 2$ in the distressing group. Indeed, this finding would be perfectly consistent with the "securitythreat compensation" purpose of attachment systems of relatedness. In classic attachment models, as well as contemporary security systems models (Hart et al. 2005), the attachment system of relatedness is activated by salient initial security threats and, in turn, resolves security threats when relatedness experiences are attained. Thus, the initial rise in security salience at $\mathrm{T} 1$ in the distressing (vs. satisfying) group may have activated attachment processes to engage in a biased motivated search that resolved salient security concerns at T2 by enhancing salient relatedness/attachment experiences in one's earliest life memories at T2. 


\section{Limitations}

Although promising, this work has important limitations. Of course, the most obvious limitation is that it is only a single study. Thus, the present study requires replication to establish the reliability of this effect. Second, this work may have neglected potential moderators of the generalization of relative need importance across time perspective. For example, we did not ask participants to indicate the domain (e.g., achievement, family/peer relationships, etc.) of the earliest autobiographical memory. Accordingly, future work could include this measure to examine whether the strength of these effects differ depending upon whether participants' "earliest autobiographical memory" occurred in an achievement domain (school) relevant to individual enhancement needs or a social/relational domain (home) more relevant to interpersonal enhancement (relatedness) or security needs. Of course, beyond these factors, this work may have neglected important individual difference factors that may also influence the generalization of relative need importance from recent to distant memories (e.g., action orientation, attachment style, or explanatory style). For example, those with insecure attachment styles may show a stronger generalization of security and relatedness needs evoked by the $\mathrm{T} 1$ distressing event recollection to their earliest autobiographical memory whereas they show no generalization of relatedness or individual enhancement needs evoked by the T1 satisfying event recollection. Future investigations should also explore cultural differences relating to self-construal (i.e., degree of individuation and separateness from others) and emotional expression related to autobiographical memories (see Zaragoza Sherman et al.2015). Accordingly, future work should examine individual difference variables and situational moderators not measured in this study.

\section{ACKNOWLEDGEMENTS}

We thank MaryAnn Kromer and Thomas Knapp for help with data collection and coding.

\section{LITERATURE CITED}

Baumeister RF, Leary MR. 1995. The need to belong: Desire for interpersonal attachments as a fundamental human motivation. Psychol Bull. 117(3):497-529.

https://doi.org/10.1037//0033-2909.117.3.497

Bowlby J. 1969. Attachment (Attachment and loss, Vol. 1). New York: Basic Books.

Bowlby J. 1973. Separation: Anxiety and anger (Attachment and loss, Vol. 2). New York: Basic Books.

Carroll PJ, Arkin RM, Seidel SD, Morris J. 2009. The relative importance of needs among traumatized and non-traumatized samples. Motiv Emotion. 33(4):373-386. https://doi. org/10.1007/s11031-009-9139-z

Carroll PJ, Wichman AL, Arkin RM. 2006. Security in the aftermath of 9-11. Basic Appl Soc Psychol. 28(4):289-290. http://dx.doi.org/10.1207/s15324834basp2804_1

Deci EL, Eghrari H, Patrick BC, Leon DR. 1994. Facilitating Internalization: The self-determination theory perspective. J Pers. 62(1):119-142. https://doi.org/10.1111/j.1467-6494.1994.tb00797.x

Deci EL, Ryan RM. 1985. Intrinsic motivation and selfdetermination in human behavior. Boston, MA: SpringerVerlag. https://doi.org/10.1007/978-1-4899-2271-7

Deci EL, Ryan RM. 2000. The 'what' and 'why' of goal pursuits: Human needs and the self-determination of behavior. Psychol Inq. 11(4):227-268. https://doi.org/10.1207/ s15327965pli1104_01

Hart J, Shaver PR, Goldenberg JL. 2005. Attachment, self-esteem, worldviews, and terror management: Evidence for a tripartite security system. J Pers Soc Psychol. 88(6): 999-1013. https:// doi.org/10.1037/0022-3514.88.6.999

Higgins ET. 1997. Beyond pleasure and pain. Am Psychol. 52(12):1280-1300. http://dx.doi.org/10.1037/0003-066X.52.12.1280

Kasser T. 2002. Sketches for a self-determination theory of values. In E.L. Deci \& R.M. Ryan (Eds.), Handbook of self-determination research (pp. 123-140). Rochester, NY: University of Rochester Press.

Maslow A. 1954. Motivation and personality. New York: Harper \& Row.

Oishi S, Diener EF, Lucas RE, Suh EM. 1999. Cross-cultural variations in predictors of life satisfaction: Perspectives from needs and values. Pers Soc Psychol Bull. 25(8):980-990. https://doi.org/10.1177/01461672992511006

Ryan RM. 1995. Psychological needs and the facilitation of integrative processes. J Pers. 63(3):397-427. https://doi. org/10.1111/j.1467-6494.1995.tb00501.x

Ryan RM, Deci EL. 2000. Self-determination theory and the facilitation of intrinsic motivation, social development, and well-being. Am Psychol. 55(1):68-78.

http://dx.doi.org/10.1037/0003-066X.55.1.68

Sheldon KM, Elliot AJ, Kim Y, KasserT. 2001. What is satisfying about satisfying events? Testing 10 candidate psychological needs. J Pers Soc Psychol. 80(2):325-339. http://dx.doi. org/10.1037/0022-3514.80.2.325

Sheldon KM, Kasser T. 2008. Psychological threat and extrinsic goal striving. Motiv Emotion. 32(1): 37-45. https://doi. org/10.1007/s11031-008-9081-5

Sheldon KM, Ryan RM, \& Reis, HT. 1996. What makes for a good day? Competence and autonomy in the day and in the person. Pers Soc Psychol Bull. 22(12):1270-1279. https:// doi.org/10.1177/01461672962212007

Wahbah MA, Bridwell LG. 1976. Maslow reconsidered: A review of research on the need hierarchy theory. Organ Beh Hum Perform. 15(2):212-240. https://doi.org/10.1016/00305073(76)90038-6

Watson D, Clark L, Tellegen A. 1988. Development and validation of brief measures of positive and negative affect: The PANAS scales. J Pers Soc Psychol. 54(6):1063-1070. http://dx.doi. org/10.1037/0022-3514.54.6.1063

Zaragoza Sherman A, Salgado S, Shao Z, Berntsen D. 2015. Life span distribution and content of positive and negative autobiographical memories across cultures. Psychol Conscious. 2(4):475-489. http://dx.doi.org/10.1037/cns0000070 